\title{
Weakly associative lattices and projective planes
}

\author{
E. Fried and Vera T. Sós
}

It is well-known how close the connection is between lattices and projective geometry. In this paper we intend to show another type of connection between a generalization of lattices and projective planes. The basic concepts of projective geometry are suppose to be known.

A weakly associative lattice is a relational system with a reflexive and antisymmetrical relation $\leqq$ such that to each pair of elements there exists both a least upper bound and a greatest lower bound. This concept has been introduced in [1] and in [4].

An important class of weakly associative lattices is the class of those which have the unique bound property. The role of this class has been described in [2] where this concept was introduced. A weakly associative lattice has the unique bound property, by definition, if each pair of elements has exactly one common upper bound and exactly one common lower bound.

DEFINITION 1. Let $\mathfrak{A}=\langle A ; \leq\rangle$ be a relational system with a reflexive and antisymmetrical relation $\leq$. Let us define $U(a)=\{x \in A ; a \leq x\}$ and $L(a)=\{y \in A$; $y \leq a\}$, for each $a \in A$. We shall call $\mathfrak{A}$ a WU-system if, for each pair $a$ and $b$ of distinct elements of $A$, both $U(a) \cap U(b)$ and $L(a) \cap L(b)$ have exactly one element denoted by $a \vee b$ and $a \wedge b$, respectively. Let, moreover, both $a \vee a$ and $a \wedge a$ denote the element $a$.

We shall use the notation $x<y$ for ' $x \leq y$ and $x \neq y$ '.

One can easily prove the following identities in any weakly associative lattice;

(i) $a \vee a=a \wedge a=a$,

(ii) $a \vee b=b \vee a$ and $a \wedge b=b \wedge a$,

(iii) $a \vee(b \wedge a)=a \wedge(b \vee a)=a$,

(iv) $[(a \wedge c) \vee(b \wedge c)] \vee c=[(a \vee c) \wedge(b \vee c)] \wedge c=c$.

Let $\mathbf{A}$ be any set. We define $W_{A}$ as follows. $W_{A}$ contains all elements of the set $\mathbf{A}$ and two more elements denoted by 0 and 1 . We define, in $W_{A}$, the following relations: $1<0$ and $0<a<1$, for all $a \in A$. Then, $W_{A}$ with the relation $x \leq y$ if and only if either $x<y$ or $x=y$ is, clearly, a WU-system.

DEFINITION 2. The WU-systems $W_{A}$ will be called singular WU-systems.

DEFINITION 3. $\mathfrak{A}=\langle A ; \leq\rangle$ will be called a regular WU-system whenever, for each $x, y \in A,|U(x)|=|U(y)|=|L(y)| .(|X|$ denotes the cardinality of the set $X$.) 
THEOREM 1. A WU-system is either singular or regular. For the proof we need the following

LEMMA. Let $\mathfrak{I}$ be $a$ WU-system and $a, b \in \mathfrak{U}$. If $a \$ b$, then $|U(a)|=|L(b)|$.

Proof. Suppose $a \ddagger b$ and let $x$ be an element of $U(a)$. We associate with $x$ the element $x \wedge b$ of $L(b)$. For $x$ and $y$ distinct elements of $U(a)$ we have $x \wedge b \neq y \wedge b$. Otherwise, from $u=x \wedge b=y \wedge b$ it follows that $a, u \in L(x) \cap L(y)$ which implies, that $a=x \wedge b \leq b$, contrary to the assumption that $a \ddagger b$. Thus, the mapping $x \rightarrow x \wedge b$ is one-to-one into $L(b)$, i.e., $|U(a)| \leqq|L(b)|$.

Now, we define $x \leq^{*} y$ by $y \leq x$. The system $\mathfrak{I}^{*}=\left\langle A ; \leq^{*}\right\rangle$ is, clearly, a WU-system. Setting $U^{*}(a)=\left\{x \in A ; a \leq^{*} x\right\}$ and $L^{*}(a)=\left\{y \in A ; y \leq^{*} a\right\}$, we have $U^{*}(a)=$ $=L(a)$ and $L^{*}(a)=U(a)$. Thus, $a \ddagger b$ implies $b \$^{*} a$, whence $|L(b)|=\left|U^{*}(b)\right| \leq$ $\leq\left|L^{*}(a)\right|=|U(a)|$. Hence $|U(a)|=|L(b)|$, and the Lemma is proved.

Proof of Theorem 1. Let $a$ and $b$ be elements of a given WU-systems $\mathfrak{A} . a \$ b$ and $b \$ a$ imply, by the Lemma, $|U(a)|=|L(b)|$ and $\mid U(a)\}=|L(a \wedge b)|=|U(b)|$. Now, let, e.g., $a<b$. We shall distinguish two cases:

(a) $x<b$ implies $x \leqq a$ and $y>a$ implies $y \geqq b$, for each $x, y$ in $\mathfrak{A}$;

$(\beta)$ there exists either an $x$ in $\mathfrak{U}$ such that $x<b$ and $x \$ a$ or a $y$ in $\mathfrak{U}$ such that $y>a$ and $y \geq b$ are valid.

In case $(\alpha)$ if $x<b$, then $x, a \in L(a) \cap L(b)$ whence $x=a$. In other words, $L(b)=$ $=\{a, b\}$. Similarly $U(a)=\{a, b\}$. Thus, for each $u \in \mathfrak{A}$, both $u \vee a$ and $u \wedge b$ belong to the set $\{a, b\}$. Thus $u \in\{u \wedge a, a, b\} \cap\{u \vee b, a, b\}$.

Hence, for $u \notin\{a, b\}$, we have $u=u \wedge a=u \vee b$, i.e., $b<u<a$, proving that $\mathfrak{A}$ is a singular WU-system.

In case $(\beta)$, we may suppose, by duality, the existence of an $x$ such that $x<b$ and $x \npreceq a$. Then, the Lemma implies $|U(b)|=|L(x)|=|U(a)|$, since $a \$ x$ and $b \$ x$. Applying this for $\mathfrak{A}^{*}$, we arrive at $|L(b)|=|L(a)|$. Hence. $\mathfrak{A}$ is a regular WU-system, for $b \$ a$ implies $|U(b)|=|L(a)|$.

THEOREM 2/A. Let $\mathfrak{X}$ be a regular WU-system. Then the elements $a \in \mathfrak{A}$ and the subsets $U(a)$ of $\mathfrak{A}$ form a projective plane whose points are the elements of $\mathfrak{A}$ and whose lines are these subsets of $\mathfrak{A}$. In addition the mapping $\varphi: a \rightarrow U(a)$ is a one-to-one mapping of the points of the plane onto the set of all lines such that a lies on $\varphi(a)$

Proof. If $a$ and $b$ are distinct elements of $\mathfrak{A}$, there exists exactly one line, namely $U(a \wedge b)$, containing both $a$ and $b$. Also $U(a) \cap U(b)$ contains only one element, namely, $a \vee b$, i.e., two different lines have exactly one common point. The regularity of $\mathfrak{A}$ implies that the cardinality of $U(a)$ does not depend on $a$. Further, the Lemma guarantees that $U(a)$ and $L(a)$ have the same cardinality, too. Hence not all points are on the same line and not all lines are on the same point. 
Finally, the mapping $\varphi: a \rightarrow U(a)$ obviously has the desired property stated in the theorem.

THEOREM 2/B. Let $P$ be a projective plane. Then, there exists a one-to-one mapping $\varphi$ of the set of its points onto the set of all its lines such that a lies on $\varphi(a)$.

Further, there exists, to any such $\psi$, a regular WU-system such that the construction described in Theorem 2/A gives us the plane $P$ and the mapping $\psi$.

Proof. First of all we are going to prove the existence of the desired mapping (which need not be unique).

We define a graph whose vertices are the disjoint union of the set of all points and of the set of all lines. Two vertices are connected in this graph if and only if one of them is a line and the other one is a point lying on the line. This is, obviously, an even graph and each vertex has the same degree. The existence of a mapping $\varphi$ in question means that this graph has a one-factor. But this is just the statement of the wellknown theorem of König (see, e.g., in [3] pp. 171 and 220).

We choose the underlying set of the WU-system to be the set of all points of $P$. Let, further, $a \leqq b$ if and only if $b$ lies on $\psi(a)$.

$a \in \psi(a)$ implies $a \leq a$. From $a \leqq b$ and $b \leqq a$ follow both $a \in \psi(b)$ and $b \in \psi(a)$. Combining this with $x \in \psi(x)$, we have that both $a$ and $b$ lie on the line $\psi(a)$ and on the line $\psi(b)$. Since distinct lines contain exactly one common point, we have either $a=b$ or $\psi(a)=\psi(b)$ which have the same meaning. Hence, the relation $\leqq$ is reflexive and antisymmetrical.

Now, let $a \leqq x$ and $b \leqq x$, for distinct $a$ and $b$. This means $x \in \psi(a) \cap \psi(b)$ proving the uniqueness of $x$. Let, dually, $y \leqq a$ and $y \leqq b$; i.e., $a, b \in \psi(y)$. Since a pair of points uniquely determine the line they lie on, $\psi(y)$ is unique. From this follows the uniqueness of $y$ since $\psi$ is one-to-one. Thus, we have a WU-system.

The mapping $a \rightarrow U(a)$ is, clearly, equal to $\psi$, hence, the theorem is proved.

COROLLARY. The regular WU-systems are equivalent to the projective planes endowed with a one-to-one mapping of the set of the points onto the set of the lines such that each point lies on its image. Since each projective plane may have more such mappings to each projective plane may belong more WU-systems.

We shall now give an example of a projective plane which yields three non-isomorphic WU-systems depending on the special choice of the one-factor of the graph in Theorem 2/B.

First, we define the following notion.

Let $x$ and $y$ be distinct elements of a WU-system $\mathfrak{A}$. We shall say that the set $\{x, y\}$ has rank 0 , whenever the set $\{x \wedge y, x \vee y\}$ is equal to the set $\{x, y\}$, i.e., $x$ and $y$ are comparable. The set $\{x, y\}$ has rank $k+1$, whenever $\{x, y\}$ does not have rank $k$ and $\{x \wedge y, x \vee y\}$ has rank $k$. 
Now, we are ready to give the example. The points of the projective plane $P$ will be the cosets modulo 13. We define, first, two relations on $P$. One can prove without any difficulty that these relations define two WU-systems $\mathfrak{U}_{1}$ and $\mathfrak{A}_{2}$.

Let $x<y$ in $\mathfrak{A}_{1}$ if and only if $y-x \in\{1,3,9\}$

Let $x<y$ in $\mathfrak{U}_{2}$ if and only if $y-x \in\{1,4,6\}$

The two projective planes must be the same, for the projective plane with 13 elements is unique. Besides, the following mapping sends the 'first' plane to the 'second' one:

$$
0 \rightarrow 0,1 \rightarrow 1,2 \rightarrow 5 \rightarrow 8 \rightarrow 12 \rightarrow 3 \rightarrow 4 \rightarrow 7 \rightarrow 11 \rightarrow 10 \rightarrow 2 ; 6 \rightarrow 9 \rightarrow 6 .
$$

Let $\varphi$ denote the mapping belonging to $\mathfrak{A}_{1}$ and we define $\psi$ as follows: $\psi(2)=$ $=\varphi(6), \psi(3)=\varphi(2), \psi(6)=\varphi(3)$, and $\psi(i)=\varphi(i)$, for $i \notin\{2,3,6\} .3 \in \varphi(2), 6 \in \varphi(3)$, $2 \in \varphi(6)$ proves that $\psi$ defines, also, a one-factor of the graph. The corresponding WU-system will be denoted by $\mathfrak{U}_{3}$.

THEOREM 3. No two of $\mathfrak{U}_{1}, \mathfrak{A}_{2}, \mathfrak{A}_{3}$ are isomorphic.

Proof. The isomorphic image of a pair of elements has, clearly, the same rank as the original one. Thus, it is enough to prove that the maximal ranks of particular pairs are different in the three examples.

The mapping $i \rightarrow i+1$ defines an isomorphism both on $\mathfrak{U}_{1}$ and on $\mathfrak{U}_{2}$. Therefore, we need only consider pairs of the form $\{0, i\}$ with $i<7$.

Firstly, we deal with $\mathfrak{A}_{1}$. The pairs $\{0,1\},\{0,3\},\{0,4\}$ have rank 0 . The other three pairs have rank 1 , since

$$
\begin{aligned}
& 0 \vee 2=3<3+9=12=0 \wedge 2, \\
& 0 \vee 5=1<1+3=4=0 \wedge 5, \\
& 0 \vee 6=9<9+1=10=0 \wedge 6 .
\end{aligned}
$$

In $\mathfrak{A}_{2}$ the pairs $\{0,1\},\{0,4\},\{0,6\}$ have rank 0 . By $0 \vee 5=6,0 \wedge 5=12=6+6$; $0 \wedge 3=12,0 \vee 3=4=12+5 ; 0 \vee 2=6,0 \wedge 2=9=6+3$ we have that the pairs $\{0,5\}$, $\{0,3\},\{0,2\}$ have rank $1,2,3$, respectively.

Finally, we consider $\mathfrak{U}_{3}$. Though the mapping $i \rightarrow i+1$ is not an isomorphism of this WU-system it will be enough to deal only with pairs of the form $\{0, i\}$.

$\psi(i)=\varphi(i)$, for $i=0,4,10,12$ imply that $\{0, j\}$ have $\operatorname{rank} 0$, for $j=1,3,4,9$, 10,12 .

It is not too hard to check:

$0 \vee 2=9<12=0 \wedge 2, \quad 0 \vee 5=1<4=0 \wedge 5, \quad 0 \vee 8=9<12=0 \wedge 8, \quad 0 \vee 11=1<10=$ $=0 \wedge 11$ proving that $\{0,2\},\{0,5\},\{0,8\},\{0,11\}$ have rank 1 . Using $3 \vee 10=11<7=$ $=3 \wedge 10$ and $3 \vee 4=5<6=3 \wedge 4$ the relations $0 \vee 6=3,0 \wedge 6=10$ and $0 \vee 7=3,0 \wedge 7=4$ one proves that both $\{0,6\}$ and $\{0,7\}$ have rank 2 . 
Thus, $\mathfrak{A}_{1}$ is not isomorphic either to $\mathfrak{U}_{2}$ or to $\mathfrak{U}_{3}$, for it does not contain pairs having rank more than 1 . In $\mathfrak{A}_{2}$, to each $x$, there exist a $y$, namely $y=x+2$, such that the rank of $\{x, y\}$ is three. Since no pair $\{0, i\}$ has rank 3 in $\mathfrak{A}_{3}, \mathfrak{U}_{3}$ is not isomorphic to $\mathfrak{A}_{2}$.

Finally, we give an effective construction of WU-systems, for projective planes over certain fields.

Let $K$ be a field and let the element $\alpha$ over $K$ have degree three. The relation $\beta \sim \gamma$ in $L=K(\alpha)$ if and only if there exists a $u \in K$ different from 0 such that $\beta=u \gamma$ is, clearly, an equivalence. Let us define a relation $\leqq$ on the set $\bar{L}$ of the classes of this equivalence such that $\beta \geqq \gamma$ means $\beta \sim \gamma(\alpha+a)$ for some $a \in K$.

THEOREM 4. $\mathfrak{U}=\langle\bar{L}, \leqq\rangle$ is a WU-system.

Proof. It is enough to deal with pairs of the form $\{1, \beta\}$, for $\bar{L}$ is a multiplicative group.

Let us consider a pair $\gamma, \delta$ with $\gamma \delta=\beta$. We shall prove, first of all, that $\gamma$ is a common upper bound of $\beta$ and 1 if and only if $\delta$ is a common lower bound of them. This is an immediate consequence of the equalities $(\gamma / \beta)=(1 / \delta)$ and $(\gamma / 1)=(\beta / \delta)$. This means, it is enough to prove that 1 and $\beta$ have a unique common upper bound. To prove uniqueness let $\gamma_{1}=(\alpha+b) \beta=u(\alpha+a)$ and $\gamma_{2}=(\alpha+d) \beta=v(\alpha+c)$. This gives us the equality $v(\alpha+b)(\alpha+c)=u(\alpha+a)(\alpha+d)$. Using that the degree of $\alpha$ over $K$ is equal to three we arrive at $u=v$ and, further, $b+c=a+d$ and $b c=a d$.

The second and the third equations yield either $b=d$ and $c=a$ or $b=a$ and $c=d$. In the first case $\gamma_{1}=\gamma_{2}$ and in the second one $\beta \sim 1$.

To prove $\beta(\alpha+a)=v(\alpha+b)$, with suitable elements for each $\beta \in L$, unless $\beta$ and 1 are comparable we must prove that the elements $v(\alpha+b) /(a+a)$ run over $L$. (In the finite case an easy computation proves this result). Incomparability means we have to deal only with elements of the form $\alpha^{2}+p \alpha+q$. Let $x^{3}+A x^{2}+B x+C$ be the monic polynomial of $\alpha$ over $K$. Thus, we have to show

$$
\left(\alpha^{2}+p \alpha+q\right)(\alpha+a)-v(\alpha+b)=\alpha^{3}+A \alpha^{2}+B \alpha+C,
$$

with suitable $a, b$ and $v \neq 0$. This yields the equations:

$$
p+a=A, \quad q+a p-v=B, \quad a q-v b=C .
$$

In case $q+a p=B$, with $a=A-p$, we have

$$
\left(a^{2}+p \alpha+q\right)(\alpha+a)-(a q-C)=\alpha^{3}+A \alpha^{2}+B \alpha+C .
$$

$a q=C$ is impossible, since the monic polynomial of $\alpha$ is irreducible and $a q-C \neq 0$ implies $\beta=1$. Thus $v=q+a p-B \neq 0$, i.e., $a=A-p, v=q+a p-B$, and $b=(a q-C) / b$ is a 
solution. This method is, obviously, not applicable for algebraically closed and real closed fields.

PROBLEMS. 1) How does the WU-system depend on the one-factor of the graph in Theorem $2 / \mathrm{B}$ ?

2) Give a generalization of Theorem $2 / B$ for projective geometries of higher dimensions.

\section{REFERENCES}

[1] E. Fried, Tournaments and nonassociative lattices, Ann. Univ. Sci. Budapest Sect. Math. 13(1970), $151-164$.

[2] E. Fried, Subdirect irreducible weekly associative lattices with congruence extension property, Ann. Univ. Sci. Budapest Sect. Math. (to a ppear).

[3] D. König, Theorie der endlichen und unendlichen Graphen, Chelsea, New York, 1950.

[4] H. Skala, Trellis theory, Algebra Universalis 1 (1971), 218-233.

[5] V. T. Sós, On graphs, fin ite geometries, and block-designs, Proc. Int. Coll. Theorie Combinatorie Acc. Naz. Lincei, Roma 1973, (to appear).

[6] H. J. Ryser, Variants of $(v, k, \lambda)$-designs, A Survey of Combinatorial Theory, North-Holland 1973.

Eötvös Loránd University

Budapest

Hungary 